\title{
Glycophorin A in two patients with congenital dyserythropoietic anemia type I and type II is partly unglycosylated ${ }^{\star}$
}

\author{
Ewa Zdebska, Monika Adamczyk-Popławska and Jerzy Kościelak ${ }^{凶}$ \\ Department of Biochemistry, Institute of Hematology and Blood Transfusion, Warszawa, Poland
}

Received: 18 July, 2000; accepted: 27 July, 2000

Key words: glycophorin A, unglycosylation, congenital dyserythropoietic anemia type I and II

\begin{abstract}
Glycophorins A from erythrocyte membranes of two patients with congenital dyserythropoietic anemia type I and type II (CDA type I and II) were analyzed for carbohydrate molar composition employing a modification of the recently published method that allowed simultaneous determination of carbohydrates and protein in electrophoretic bands of glycoproteins separated by sodium dodecyl sulfatepolyacrylamide gel electrophoresis (Zdebska \& Kościelak, 1999, Anal. Biochem., 275, 171-179). The modification involved a preliminary extraction of erythrocyte membranes with aqueous phenol, subsequent electrophoresis and analysis of the extracted glycophorins rather than electrophoresis and analysis of the glycophorin from intact erythrocyte membranes. The results showed a large deficit of $\mathrm{N}$-acetylgalactosamine, galactose, and sialic acid residues in glycophorin A from patients with CDA type I and type II amounting to about $45 \%$ and $55 \%$, respectively. The results strongly suggest that glycophorin $A$ in these patients is partly unglycosylated with respect to O-linked glycans. In addition, glycophorin A from erythrocytes of a patient with CDA II but not CDA I exhibited a significant deficit of mannose and $N$-acetylglucosamine suggesting that its N-glycosylation site was also partly unglycosylated.
\end{abstract}

Congenital dyserythropoietic anemias (CDAs) are a group of rare hereditary diseases that are characterized by ineffective erythropoiesis and a certain degree of multinuclearity of erythroblasts (Wickramasinghe,
1998; 2000). Three principal types of the disease, differing in morphology and ultrastructure of erythroblasts as well as clinical symptoms, have been identified (Heimpel \& Wendt, 1968). Erythrocytes from type II pa-

\footnotetext{
$\star^{\star} 75$ th Anniversary of Membrane Lipid Bilayer Concept.

${ }^{\star}$ To whom correspondence should be addressed: Prof. Jerzy Kościelak, Department of Biochemistry, Institute of Hematology and Blood Transfusion, Chocimska 5, 00-957 Warszawa, Poland; tel/fax: (48 22) 848 9515; fax: (48 22) 848 8970; e-mail: kosci@atos.warman.com.pl

Abbreviations: CDA, congenital dyserythropoietic anemia; CHO, carbohydrates; GPA, glycophorin A; HEMPAS, hereditary erythroblast multinuclearity with positive acidified serum test; GalNAc, $N$-acetylgalactosamine; GlcNAc, $N$-acetylglucosamine; NeuAc, $N$-acetylneuraminic acid
} 
tients are in addition lysed by acidified sera from about $30 \%$ of $\mathrm{AB} 0$ compatible donors, hence another name for type II of the disease is Hereditary Erythroblast Multinuclearity with Positive Acidified Serum test (acronym HEMPAS) (Crookston et al., 1969). HEMPAS has attracted most attention because of glycoconjugate abnormalities in erythrocyte membranes consisting of hypoglycosylation of erythrocyte anion transporter, also known as band 3 glycoprotein, and accumulation of certain glycosphingolipids including lactotriaosylceramide, neolactotetraosylceramide and polyglycosylceramides (Fukuda, 1999). Recently, however, we have found that erythrocytes from a patient with CDA type I exhibited similar, though less pronounced, glycoconjugate abnormalities as those from patients with HEMPAS (Zdebska \& Kościelak, 1999). Hypoglycosylation of erythrocyte band 3 in that study was demonstrated employing our new method of determination of carbohydrate molar composition of glycoproteins separated by SDS/PAGE (Zdebska \& Kościelak, 1999). Using a similar approach we report here that glycoconjugate abnormalities in erythrocytes from both CDA type I and II patients concern also glycophorin A (GPA), a glycoprotein of $31 \mathrm{kDa}$ that contains about 12 O-linked glycans (Zdebska \& Kościelak, 1999) (out of 16 potential O-glycosylation sites) (Pisano et al. 1993) and a single N-linked glycan. This glycoprotein is a major carrier of erythrocyte sialic acid and migrates in SDS/PAGE mainly as a dimer of about $80 \mathrm{kDa}$ with a trivial name of PAS-1. Unfortunately, unlike band 3 that migrates in SDS/PAGE as an almost pure substance, PAS- 1 is heavily contaminated with other glycoproteins and proteins (Zdebska \& Kościelak, 1999). Therefore, we had to modify our previous method of analysis of PAS-1 so as to isolate it in a sufficient state of purity. We have achieved this by preliminary extraction of erythrocyte membranes with aqueous phenol followed by SDS/PAGE of the GPA enriched extract. Thus, this paper presents both a new tech- nique and its immediate application. Previously, Mawby et al. (1983) reported that sialic acid, galactose and $N$-acetylglucosamine, but not $\mathrm{N}$-acetylgalactosamine, contents of glycophorin A in erythrocyte membranes from CDA II patients were reduced. More recently, Tomita \& Parker (1994) performed an electrophoretic study of radioimmunoprecipitated CDA II glycophorin A that was subjected to the action of peptide- $N$-glycosidase $\mathrm{F}$ and O-glycanase. They concluded that it was abnormally glycosylated with respect to O-linked glycans, possibly of lactosaminoglycan nature.

\section{PATIENTS AND METHODS}

Patients. Our CDA type I blood was drawn from a recently diagnosed patient K.R. with blood group 0 (Zdebska et al., 2000b) whereas blood of patient L.F. with HEMPAS (also of blood group 0) was obtained through the courtesy of Prof. H. Schachter. Incidentally, patient L.F. was among the first five patients with CDA type II initially described by Crookston et al. (1969). Erythrocyte membranes were obtained by the method of Dodge et al. (1963). The membranes were freezedried and extracted with aqueous phenol according to Lisowska et al. (1987). Briefly, erythrocyte membranes (5-10 mg) were suspended in $0.5-1.0 \mathrm{ml}$ of $0.9 \% \mathrm{NaCl}$, treated with an equal volume of $90 \%$ phenol, mixed, and heated at $65^{\circ} \mathrm{C}$ for $30 \mathrm{~min}$. The emulsion was separated into two phases by freezing at $-20^{\circ} \mathrm{C}$, and the upper phase, containing glycophorins A, B, and C was dialyzed for 2 days, centrifuged at $16000 \times \mathbf{g}$ and freezedried. The dry material (about $10 \mu \mathrm{g}$ ), further referred to as crude GPA, was dissolved in electrophoresis buffer and subjected to SDS/PAGE (Zdebska \& Kościelak, 1999). The separated bands were electroblotted onto polivinylidene difluoride membrane (Millipore, Bedford, MA, U.S.A.), the region of PAS-1 was excised and subjected in situ to se- 
quential hydrolysis with $0.2 \mathrm{M}$ trifluoroacetic acid (TFA), $2 \mathrm{M}$ TFA and lastly with $6 \mathrm{M} \mathrm{HCl}$ to release sialic acid, neutral sugars with hexosamines, and amino acids, respectively, exactly as described (Zdebska \& Kościelak, 1999). Carbohydrates were quantitated by High pH Anion Exchange Chromatography with Pulsed Amperometric Detection employing a Dionex Series 4500i system, a Carbopac PA-1 column $(4 \mathrm{~mm} \times 250 \mathrm{~mm})$ and a Carbopac PA-1 guard column $(4 \mathrm{~mm} \times 40 \mathrm{~mm})$. Amino acids were determined by the t-test for unpaired samples. A highly purified glycophorin A standard was a gift from Prof. W. Dahr.

\section{RESULTS}

The results are presented in Table 1. The upper portion of Table 1 shows carbohydrate molar and weight \% composition of three preparations of glycophorin A that were obtained either by our previously published procedure

Table 1. Molar and weight percentage composition of different preparations of glycophorin A (GPA)

\begin{tabular}{|c|c|c|c|c|c|c|c|c|c|}
\hline \multirow{2}{*}{$\begin{array}{l}\text { Material analyzed } \\
\text { and blood group }\end{array}$} & Fuc & GalNAc & GlcNAc & Gal & & Man & NeuAc & Total CHO & Protein \\
\hline & \multicolumn{9}{|c|}{$\mathrm{mol} / \mathrm{mol} ; \%$} \\
\hline \multicolumn{10}{|l|}{ GPA, $0^{\mathrm{a}}$} \\
\hline $\mathrm{n}=1$ & $2.1 ; 1.1$ & $6.4 ; 4.5$ & $6.7 ; 4.7$ & 14.9 & 8.5 & $3.0 ; 1.7$ & $12.6 ; 12.4$ & $45.7 ; 32.9$ & $1.0 ; 67.1$ \\
\hline \multicolumn{10}{|l|}{ GPA, $0^{b}$} \\
\hline $\mathrm{n}=1$ & $2.7 ; 1.4$ & $10.8 ; 7.6$ & $5.4 ; 3.8$ & 24.5 & 14.0 & $3.0 ; 1.7$ & $21.8 ; 21.4$ & $68.2 ; 49.9$ & $1.0 ; 50.1$ \\
\hline \multicolumn{10}{|l|}{ GPA, Standard ${ }^{\mathrm{c}}$} \\
\hline \multicolumn{10}{|l|}{$\mathrm{GPA}, 0 \mathrm{M}^{\mathrm{b}}$} \\
\hline $\mathrm{n}=4$ & $2.7 ; 1.4$ & $11.2 ; 7.9$ & $6.3 ; 4.4$ & 23.9 & 13.7 & $3.0 ; 1.7$ & $20.9 ; 20.5$ & $68.1 ; 49.6$ & $1.0 ; 50.4$ \\
\hline S.D. \pm & $0.3 ; 0.2$ & $0.9 ; 0.6$ & $0.9 ; 0.6$ & 0.5 & 0.3 & $0.3 ; 0.2$ & $1.2 ; \quad 1.2$ & $1.5 ; \quad 1.2$ & \\
\hline \multicolumn{10}{|l|}{$\mathrm{GPA}, 0 \mathrm{~N}^{\mathrm{b}}$} \\
\hline $\mathrm{n}=3$ & $3.0 ; 1.6$ & $13.2 ; 9.3$ & $7.5 ; 5.2$ & 23.5 & 13.4 & $2.9 ; 1.6$ & $20.8 ; 20.4$ & $70.8 ; 51.5$ & \\
\hline S.D. \pm & $0.6 ; 0.3$ & $1.3 ; 0.9$ & $0.6 ; 0.4$ & 1.3; & 0.7 & $0.3 ; 0.2$ & $0.7 ; \quad 0.7$ & $0.5 ; \quad 0.9$ & $1.0 ; 48.9$ \\
\hline \multicolumn{10}{|l|}{ GPA, $A^{b}$} \\
\hline \multicolumn{10}{|l|}{ GPA, $\mathrm{B}^{\mathrm{b}}$} \\
\hline \multicolumn{10}{|l|}{ GPA, CDA I $^{\mathrm{b}}$} \\
\hline $\begin{array}{l}\text { GPA, CDA II } \\
n=1\end{array}$ & $1.0 ; 0.5$ & $5.6 ; 3.9$ & $4.0 ; 2.8$ & 9.5 & 5.4 & $2.3 ; 1.3$ & $10.3 ; 10.1$ & $32.5 ; 24.0$ & $1.0 ; 76.0$ \\
\hline
\end{tabular}

${ }^{a}$ Erythrocyte membranes, electrophoresed and electroblotted; ${ }^{b}$ subjected to preliminary extraction with aqueous phenol, then electrophoresed and electroblotted; ${ }^{c}$ highly purified GPA standard, additionally electrophoresed and electroblotted.

fluorescamine method (Undefriend et al., 1972) using a Perkin Elmer LS50 spectrofluorimeter. The same instrument was used for determination of sphingosine by the method of Higgins (1984). All determinations were made in duplicate. Statistical comparisons were made with the use of Student's
(Zdebska \& Kościelak, 1999), or by the modified method employing preliminary extraction of erythrocyte membranes with aqueous phenol (see under Patients and Methods). It is evident that the extraction with aqueous phenol in combination with SDS/PAGE is highly effective in removing protein and glyco- 
protein impurities from PAS-1. The absence of sphingolipid impurities in PAS-1 purified by this method was demonstrated by the lack of sphingosine (0.0 weight \%) among its hydrolytic products. Sphingosine content of crude GPA amounted to 9.7 weight \%. It should be pointed out that PAS-1 obtained by the modified procedure has exactly the same carbohydrate molar composition as our highly purified glycophorin standard and by this criterion is $100 \%$ pure. Thus, PAS- 1 from phenol extracted erythrocyte membranes will be further referred to as GPA. In view of the reports that glycopeptides isolated from GPA from $0 \mathrm{~N}$ erythrocytes have some excess of unsubstituted $\mathrm{N}$-acetylgalactosamine over those from 0M erythrocytes (Krotkiewski et al., 1997) and that GPA from 0 erythrocytes has much less carbohydrates than that from A or B erythrocytes (Krotkiewska et al., 1999) we evaluated also the effect of these blood groups on carbohydrate molar composition of GPA as determined by the present method. As shown in Table 1 we found that GPA from $0 \mathrm{~N}$ erythrocytes has indeed more of total $N$-acetylgalactosamine than that from $0 \mathrm{M}$ erythrocytes though, due to a small number of samples analyzed, the difference is barely significant statistically $(P=0.06)$. On the other hand, carbohydrate molar composition of GPA was very much the same regardless of its AB0 blood group status.

The carbohydrate molar composition of GPAs from erythrocytes of patients with CDA I and CDA II is presented in the bottom part of Table 1. A clear reduction of carbohydrate content including components of O-linked glycans: $N$-acetylgalactosamine, galactose, and sialic acid is seen for both preparations though is slightly more pronounced in GPA from CDA type II erythrocytes. This reduction is far too large to be accounted for by a possible effect of blood group phenotype on carbohydrate molar composition of glycophorins. We also found that mannose and $N$-acetylglucosamine contents of GPA from CDA type II but not CDA type I were reduced.

\section{DISCUSSION}

The results clearly show that glycoprotein abnormalities in CDA are not restricted to band 3 and glycosphingolipids but involve also GPA. In the case of the latter glycoprotein, O-linked glycosylation sites seem to be partly unglycosylated as suggested by the significantly reduced numbers of $\mathrm{N}$-acetylgalactosamine residues in glycophorins from both CDA type I and CDA type II patients. This finding is reported for the first time. We use the term unglycosylation for a situation when a glycan is missing in its entirety and the term hypoglycosylation to describe a glycan with fewer carbohydrates at the non-reducing terminus but a complete inner core that links the glycan to the protein moiety (Zdebska et al., 2000a). When structural information is inadequate we use the traditional term underglycosylation. The partial absence of O-linked glycans in GPA is reflected by decreased numbers not only of $\mathrm{N}$-acetylgalactosamine residues but also of galactose and sialic acid. Thus, unglycosylation involves glycans with carbohydrate composition that is typical for O-linked chains of GPA. In addition, we observed significantly reduced mannose and $N$-acetylglucosamine contents in GPA from CDA type II erythrocytes suggesting that also the N-linked glycan might be partly unglycosylated. This is also described for the first time. In addition, we measured total sialic acid in GPA from patient L.F. and found it to be decreased by $14 \%(P=$ $0.006)$, a value very similar to those reported by Mawby et al. (1983).

The mechanism of glycoconjugate abnormalities affecting band 3 and glycosphingolipids in CDA is unknown. Previously, underglycosylation of band 3 in CDA type II was regarded as a cause of dyserythropoiesis on the assumption that carbohydrate-deficient, poorly soluble in water, and aggregationprone band 3 would disorganize membrane structure and interfere with normal cytokinesis of erythroblasts (Fukuda, 1990; 
1999). Evidence was presented that the hypoglycosylation of band 3 was due to a deficiency of either $\beta$-4-galactosyltransferase, $\mathrm{N}$-acetylglucosaminyltransferase II, or mannosidase II. More recently it has been found, however, that the CDA II gene localizes to a different chromosome than the genes for the aforementioned enzymes (Gasparini et al., 1997) and in another study they were excluded as candidate genes for the disease (Iolascon et al., 1997). We found that glycoconjugate abnormalities in erythrocytes from patients with CDA type I and CDA type II were basically similar though less pronounced in CDA type I with hypoglycosylation of band 3 being relatively mild (Zdebska et al., 2000b). Thus, in our opinion, the described glycoconjugate abnormalities are not the cause of dyserythropoiesis but, together with the abnormal composition of glycosphingolipids, is rather a consequence of dyserythropoiesis and the associated disturbance of the cell cycle as documented for all three types of CDA (Wickramasinghe, 1998). The presently described partial unglycosylation of glycophorin A may result from a similar cause. Interestingly, hypoglycosylation of band 3 and partial unglycosylation of glycophorin A was found by us also in erythrocytes from patients with congenital disorder of glycosylation (CDG) type 1a (Zdebska et al., 2000b) caused by a deficiency of phosphomannomutase (Carchon et al., 1999). The latter disease is one of congenital disorders of glycosylation that until recently were known under the name of carbohydrate deficient glycoprotein syndromes (Aebi et al., 2000). A simultaneous disturbance of glycosylation of the two most abundant glycoproteins of erythrocyte membranes in two different diseases raises an issue of their postulated partnership during biosynthesis (Poole, 2000). Thus, the glycan of band 3 is larger when glycophorin A in erythrocytes is missing (Bruce et al., 1994; Gahmberg et al., 1976; Tanner et al., 1976) and smaller when the expression of the glycophorin is increased (Dahr et al., 1987). Moreover, erythrocytes of mice with targeted inactivation of the band 3 gene were deficient in glycophorin A and a chaperone function of band 3 with respect to the biosynthesis of GPA was predicted (Hassoun et al., 1998). On the other hand, the biosynthesis of GPA in human erythroblasts precedes that of band 3 , so a "chaperone" function of band 3 with respect to GPA during biosynthesis is unlikely in man (Daniels, 1999). Thus, the apparently quite similar glycoconjugate abnormalities affecting both glycophorin A and band 3 in CDG1a and CDA type I and type II cannot be adequately explained at present.

Lastly we would like to comment on the effect of MN and $\mathrm{AB} 0$ blood groups on carbohydrate molar composition of GPA. In the first instance it looks as if total $N$-acetylgalactosamine content of GPA from $0 \mathrm{~N}$ erythrocytes is higher than that from $0 \mathrm{M}$ erythrocytes. These additional $N$-acetylgalactosamine residues in GPA from $0 \mathrm{~N}$ erythrocytes are probably not substituted with either sialic acid or galactose since the total contents of the two latter sugars in GPAs from $0 \mathrm{M}$ and $0 \mathrm{~N}$ erythrocytes are identical. The number of samples analyzed was, however, too small to draw definite conclusions which were not the aim of this paper. We only wanted to know whether the blood group phenotype could significantly affect our results on carbohydrate molar composition of CDA type I and II erythrocytes. This is obviously not the case. In another report, however, the differences found by Krotkiewska et al. (1999) were very large with GPA from 0 erythrocytes having $40 \%$ less of total carbohydrates than that from A or B erythrocytes. So even with only a limited number of samples analyzed we conclude that our results do not confirm those findings. Yet we do not exclude the effect of blood group phenotype on carbohydrate molar composition of glycophorins but only question is its magnitude. 


\section{R E F E R E N C E S}

Aebi, M., Barone, R., Berger, E.G., Bjursell, C., Briones, P., Cardo, E., Clayton, P., Cormier-Daire, V., Cuer, M., de Koning, T., de Lonay, P., de Loos, F., Dorland, L., Dupre, T., Fabritz, L., Fiumara, A., Freeze, H., Grunewald, S., Hasilik, M., Helenius, A., Hennet, T., Heykants, L., Imbach, T., Jaeken, J., Keir, G., Kjaergaard, S., Klein, A., Kościelak, J., Körner, C., Krasnewich, D., Kupers, L., Lehle, L., Lubke, T., Marquardt, T., Matthijs, G., Niehues, R., Peters, V., Raab, Roussel, P.M., Saether, O., Seta, N., Schachter, H., Schenk, B., Schollen, E., Schwartz, M., Skovby, F., Stutz, A., Tamashita, K., Thiel, C., Uller, A., van Schaftingen, E., Verbert, A., von Figura, K., Vilaseca, A., Wahlstrom, J.G., Wevers, R. \& Winchester, B. (2000) Carbohydrate-deficient glycoprotein syndromes become congenital disorders of glycosylation: An updated nomenclature for CDG. Glycobiology 10, Glycoconjugate J., 16 (in press).

Bruce, L.J., Groves, J.D., Okubo, Y., Thilaganathan, B. \& Tanner, M.J.A. (1994) Altered band 3 structure and function in glycophorin A- and B-deficient (MkMk) red blood cells. Blood 84, 916-922.

Carchon, H., Van Schaftingen, E., Matthijs, G. \& Jaeken, J. (1999) Carbohydrate-deficient glycoprotein syndrome type IA (phosphomannomutase-deficiency). Biochim. Biophys. Acta 1455, 155-165.

Crookston, J.H., Crookston, M.C., Brunie, K.L., Francombe, W.H., Dacie, J.V., Davis, J.A. \& Lewis, S.M. (1969) Hereditary erythroblastic multinuclearity associated with a positive acidified-serum test: A type of congenital dyserythropoietic anaemia. Br. J. Haematol. 17, 11-26.

Dahr, W., Moulds, J., Unger, P. \& Kordowicz, M. (1987) The Dantu erythrocyte phenotype of the NE variety. I. Dodecylsulfate polyacrylamide gel electrophoresis studies. Blut 55, 1931.

Daniels, G. (1999) Fuctional aspects of red cell antigens. Blood Rev. 13, 14-35.

Dodge, J.T., Mitchell, C. \& Hanahan, D.J. (1963) The preparation and chemical characteristics of hemoglobin-free ghosts of human erythrocytes. Archiv. Biochem. Biophys. 100, 119130.

Fukuda, M.N. (1990) HEMPAS disease: Genetic defect of glycosylation. Glycobiology 1, 9-15.

Fukuda, M.N. (1999) HEMPAS. Biochim. Biophys. Acta 1455, 231-239.

Gahmberg, C.G., Myllyla, G., Leikola, J., Pirkola, A. \& Nordling, S. (1976) Absence of the major sialoglycoprotein in the membrane of human En(a-) erythrocytes and increased glycosylation of band 3. J. Biol. Chem. 251, 61086116.

Gasparini, P., del Giudice, E.M., Delaunay, J., Totaro, A., Granatiero, M., Merchionda, S., Zalante, L. \& Iolascon, A. (1997) Localization of the congenital dyserythropoietic anemia II locus to chromosome $20 \mathrm{q} 11.2$ by genomewide search. Am. J. Hum. Genet. 61, 1112-1116.

Hassoun, H., Hanada, T. \& Lutchman, M (1998) Complete deficiency of glycophorin A in red blood cells from mice with targeted inactivation of the band 3 (AE1) gene. Blood 91, $2146-2151$.

Heimpel, H. \& Wendt, F. (1968) Congenital dyserythropoietic anaemia with karyorrhexis and multinuclearity of erythroblasts. Hel. Med. Acta 34, 103-115.

Higgins, T.J. (1984) Simplified fluorometric assay for sphingosine bases. J. Lipid Res. 25, 1007-1009.

Iolascon, A., del Giodice, E.M., Perrotta, S., Granatiero, M., Zelante, L. \& Gasparini, P. (1997) Exclusion of three candidate genes as determinants of congenital dyserythropoietic anemia type II (CDA-II). Blood 90, 4197-4200.

Krotkiewska, B., Zwierz, K. \& Krotkiewski, H. (1999) The carbohydrate moiety of human glycophorin in CDG syndrome. Acta Biochim. Polon. 46, 371-376.

Krotkiewski, H., Duk, M., Syper, D., Lis, H., Sharon, N. \& Lisowska, E. (1997) Blood group $\mathrm{MN}$-dependent difference in degree of galactosylation of O-glycans of glycophorin $\mathrm{A}$ is restricted to the GalNAc residues located on 
amino acid residues 2-4 of the glycophorin polypeptide chain. FEBS Lett. 406, 296-300.

Lisowska, E., Messeter, L., Duk, M., Czerwiński, M. \& Lundblad, A. (1987) A monoclonal anti-glycophorin A antibody recognizing the blood M determinant: Studies on the subspecificity. Mol. Immunol. 24, 605-614.

Mawby, W.J., Tanner, M.J.A., Anstee, D.J. \& Clamp, J.R (1983) Incomplete glycosylation of erythrocyte membrane proteins in congenital dyserythropoietic anaemia type II (CDA II). Br. J. Haematol. 55, 357-368.

Pisano, A., Redmond, J.W., Williams, K.R. \& Gooley, A.A (1993) Glycosylation sites identified by solid-phase Edman degradation: O-linked glycosylation motifs on human glycophorin A. Glycobiology 3, 429-435.

Poole, J. (2000) Red cell antigens on band 3 and glycophorin A. Blood Rev. 14, 31-43.

Tanner, M.J.A., Jenkins, R.E., Anstee, D.J. \& Clamp, J.R. (1976) Abnormal carbohydrate composition of the major membrane penetrating protein of $\operatorname{En}(\mathrm{a}-)$ human erythrocytes. Biochem. J. 155, 701-703.

Tomita, A. \& Parker, Ch.J. (1994) Aberrant regulation of complement by the erythrocytes of hereditary erythroblastic multinuclearity with a positive acidified serum lysis test (HEMPAS). Blood 83, 250-259.
Undefriend, S., Stein, S., Bohlen, P., Dairman, W., Leimbgruber, W. \& Weigele, M. (1972) Fluorescamine: A reagent for assay of amino acids, peptides, proteins, and primary amines in the picomole range. Science 178, 871-872.

Wickramasinghe, S.N. (1998) Congenital dyserythropoietic anaemias: Clinical features, haematological morphology an new biochemical data. Blood Rev. 12, 178-200.

Wickramasinghe, S.N. (2000) Congenital dyserythropoietic anemias. Curr. Opin. Hematol. 7, $71-78$.

Zdebska, E. \& Kościelak, J. (1999) A single-sample method for determination of carbohydrate and protein contents in glycoprotein bands separated by sodium dodecyl sulfatepolyacrylamide gel electrophoresis. Anal. Biochem. 275, 171-179.

Zdebska, E., Jaeken, J. \& Kościelak, J. (2000a) Band 3 glycoprotein and glycophorin A from erythrocytes of children with CDG Ia are underglycosylated. Electrophoresis (in press).

Zdebska, E., Woźniewicz, B., Adamowicz-Salach, A. \& Kościelak, J. (2000b) Erythrocyte membranes from a patient with congenital dyserythropoietic anaemia type I (CDA-I) show identical, though less pronounced, glycoconjugate abnormalities to those from patients with CDA-II (HEMPAS). Br. J. Haematol. (in press). 